\title{
BMI open Effect of bile acid sequestrants on glycaemic control: protocol for a systematic review with meta-analysis of randomised controlled trials
}

\author{
Morten Hansen, David Peick Sonne, Kristian Hallundbæk Mikkelsen, \\ Lise Lotte Gluud, Tina Vilsbøll, Filip Krag Knop
}

To cite: Hansen M, Sonne DP, Mikkelsen KHæk, et al. Effect of bile acid sequestrants on glycaemic control: protocol for a systematic review with meta-analysis of randomised controlled trials. BMJ Open 2012;2:e001803.

doi:10.1136/bmjopen-2012001803

- Prepublication history and additional material for this paper are available online. To view these files please visit the journal online (http://dx.doi.org/10.1136/ bmjopen-2012-001803).

Received 11 July 2012 Accepted 2 October 2012

This final article is available for use under the terms of the Creative Commons Attribution Non-Commercial 2.0 Licence; see http://bmjopen.bmj.com

Diabetes Research Division, Department of Internal Medicine, Gentofte Hospital, University of Copenhagen, Hellerup, Denmark

Correspondence to Filip K Knop; filipknop@ dadlnet.dk

\section{ABSTRACT}

Introduction: In addition to the lipid-lowering effect of bile acid sequestrants (BASs), they also lower blood glucose and, therefore, could be beneficial in the treatment of patients with type 2 diabetes mellitus (T2DM). Three oral BASs are approved by the US Food and Drug Administration (FDA) for the treatment of hypercholesterolaemia: colestipol, cholestyramine and colesevelam. The BAS colestimide/colestilan is used in Japan. Colesevelam was recently approved by the FDA for the treatment of T2DM. We plan to provide a systematic review with meta-analysis of the glucoselowering effect of BASs with the aim to evaluate their potential as glucose-lowering agents in patients with T2DM.

Methods and analysis: In accordance with the preferred reporting items for systematic reviews and meta-analyses statement, a systematic review with meta-analysis of randomised clinical trials of BASs (vs placebo, oral antidiabetes drugs or insulin), reporting measures of glycaemic control in adult patients with T2DM, will be performed. Change in glycated haemoglobin constitutes the primary endpoint, and secondary endpoints include changes in fasting plasma glucose, low-density lipoprotein cholesterol, highdensity lipoprotein cholesterol, total cholesterol, triglycerides, body weight and body mass index and adverse events. Electronic searches will be performed in The Cochrane Library, MEDLINE and EMBASE, along with manual searches in the reference lists of relevant papers. The analyses will be performed based on individual patient data and summarised data. The primary meta-analysis will be performed using random effects models owing to expected intertrial heterogeneity. Dichotomous data will be analysed using risk difference and continuous data using weighted mean differences, both with $95 \% \mathrm{Cls}$.

Ethics and dissemination: The study will evaluate the potential of BASs as glucose-lowering agents and possibly contribute to the clinical management of patients with T2DM.

Results: The study will be disseminated by peerreview publication and conference presentation.

Protocol registration: PROSPERO CRD42012002552.

\section{ARTICLE SUMMARY}

Article focus

- Impact of bile acid sequestrants (BASs) on glycaemic control (HbA1c), effects on fasting plasma glucose, body weight (and body mass index, BMI), lipids and adverse events in patients with type 2 diabetes mellitus (T2DM).

Key messages

- A systematic review with meta-analysis on the glucose-lowering effect of BASs is lacking, but at the same time needed to evaluate the potential of BASs as glucose-lowering agents in patients with T2DM. There is an unmet need for effective, individualised and safe treatment, as only a small fraction of the patients with type 2 diabetes reach the treatment goals.

Strengths and limitations of this study

- A clear strength is the knowledge and experience, within our group - on how to conduct a systematic review and meta-analysis. A possible weakness may be the quality of the trials we identify.

\section{INTRODUCTION}

\section{Description of the condition}

Type 2 diabetes mellitus (T2DM) is a severe metabolic disease characterised by relative insulin deficiency, including defective insulin secretion and insulin resistance, inappropriate glucagon secretion and impaired incretin effect resulting in fasting and postprandial hyperglycaemia. ${ }^{2}$ T2DM is associated with overweight and dyslipidaemia and increases long-term risk of microvascular and macrovascular disease. ${ }^{3}$

\section{Description of the intervention}

In recent years, it has become clear that bile acids are not only simple fat solubilisers, but also signalling molecules that play an important role in lipid, glucose and energy 
metabolism. ${ }^{4-6}$ In line with this, clinical studies have shown that bile acid sequestrants (BASs) in addition to their well-established lipid-lowering effects ${ }^{7-9}$ can lower blood glucose and, therefore, could be potentially beneficial in the treatment of patients with T2DM. ${ }^{10-12}$ BASs, also known as resins, are large, non-absorbable, polymer molecules that bind negatively charged bile salts in the intestine. This diverts bile acids from the enterohepatic cycle and increases their faecal excretion. ${ }^{13}$ The end result is increased bile acid (and cholesterol) synthesis with upregulation of the low-density lipoprotein (LDL) receptors. Four oral BASs are available for the treatment of LDL-hypercholesterolaemia: colestipol, cholestyramine, colestilan/colestimide (in Japan) and colesevelam. In 2008, colesevelam was approved by the US Food and Drug Administration-based on three large pivotal studies ${ }^{10-12}$ - for the treatment of hyperglycaemia in T2DM.

\section{How the intervention might work}

The mechanism(s) by which BASs exert their glucoselowering action is not completely understood. Data from in vitro and in vivo animal and human studies have suggested different mechanisms including enhanced glucose-stimulated release of the incretin hormone glucagon-like peptide-1 (GLP-1) ${ }^{14-18}$ and activation of the nuclear Farnesoid X receptor, which is implicated in lipid and glucose metabolism. ${ }^{6}{ }^{19}$ It has been speculated that increased GLP-1 secretion induced by BASs may be dependent on increased concentration of bile acids in the lumen of the gut and subsequent bile acid-mediated activation of the seven-transmembrane receptor TGR5 present in GLP-1-secreting enteroendocrine L cells, ${ }^{20} 21$ thereby explaining their glucose-lowering effect. ${ }^{22}$ The glucose-lowering actions of GLP-1 are mediated by glucose-stimulated insulin secretion, inhibition of glucagon secretion and a suppressive effect on appetite and food intake. ${ }^{23}$ Also, although studies are conflicting, ${ }^{24} 25$ it is believed that the disturbed glucose homeostasis in diabetes is associated with changes in bile acid pool size and composition. ${ }^{6} 1326$

\section{Why is it important to do this review?}

T2DM affects more than 300 million people worldwide, according to the WHO. ${ }^{27}$ High level of glycated haemoglobin (HbAlc) is an established predictor of cardiovascular disease in patients with T2DM. ${ }^{28-30}$ However, recent large clinical trials have shown that intensive treatment (resulting in HbAlc of $\leq 6.0 \%$ ) in longstanding T2DM might be harmful, ${ }^{31-33}$ and, thus, individualised glycaemic control is pivotal in reducing morbidity and mortality of T2DM. ${ }^{34} 35$ Hyperlipidaemia is an important part of the pathophysiology of T2DM, and treatment with lipid-lowering drugs reduces cardiovascular mortality in T2DM. ${ }^{3}$ However, despite numerous glucose and lipid-lowering agents being used in the management of patients with T2DM, there is still an unmet need for effective, individualised and safe treatment, as only a small fraction of the patients reach the treatment goals. ${ }^{36} 37$ To our knowledge, a systematic review with meta-analysis on the glucose-lowering effect of BASs is lacking, but at the same time it is needed to evaluate the potential of BASs as glucose-lowering agents in patients with T2DM.

\section{OBJECTIVES}

The primary objective of the present protocol is to evaluate the impact of BASs on glycaemic control (HbAlc), and secondary objectives include effects on fasting plasma glucose, body weight (and body mass index $(\mathrm{BMI})$ ) and lipids and adverse events associated with the use of BASs in patients with T2DM.

\section{METHODS}

The review will be performed according to the recommendations specified in the Cochrane Handbook for Intervention Reviews. ${ }^{38}$ The reporting of the review will follow the preferred reporting items for systematic reviews and meta-analyses statement. ${ }^{39}$ The analyses will be performed based on analyses of individual patient data from published randomised trials and summarised data presented in published trials or supplied by authors of included trials.

\section{Criteria for considering studies for this review Types of studies}

The review will include randomised controlled trials, irrespective of blinding, publication status or language. The first period of any crossover trials will be included. Unpublished trials will be included if the methodology and data are accessible in written form.

\section{Types of participants}

Adult patients (at least 18 years of age) of both genders with T2DM will be included. Inclusion criteria should be reported in the included trials. Ideally, the diagnostic criteria for T2DM should be based on the criteria of the WHO, the American Diabetes Association and/or the European Association for the Study of Diabetes, ${ }^{37}{ }^{40}$ but if necessary, trials will be included with the definition of T2DM used by the authors of the trial in question.

\section{Types of interventions}

The intervention comparisons will include BASs (cholestyramine, colestilan/colestimide, colestipol or colesevelam) versus placebo, oral antidiabetic drugs or insulin. Co-interventions with other antidiabetic agents will be accepted if administered to the intervention and control group.

\section{Types of outcome measures}

The following outcome measures will be assessed based on analyses of individual patient data from included trials or from published reports. 
Primary outcome measure

- HbAlc

\section{Secondary outcome measures}

- Fasting plasma glucose

- LDL cholesterol, high-density lipoprotein cholesterol, total cholesterol and triglycerides

- Body weight and BMI

- Adverse events (defined based on the international guidelines for good clinical practice as any untoward medical occurrence)

\section{Search methods for identification of studies}

\section{Electronic searches}

The electronic searches will be performed in The Cochrane Library, MEDLINE and EMBASE using the following strategy:

- Cochrane Library ('bile acid sequestrants' OR 'sequestrants') AND 'type 2 diabetes'

- Medline (1) exp type 2 diabetes, bile acid sequestrants; (2) sequestrants.mp [mp=title, original title, abstract, name of substance word, subject heading word and unique identifier]

- Embase (1) exp bile acid sequestrants/; (2) sequestrants.mp [mp=title, abstract, subject headings, heading word, drug trade name, original title, device manufacturer and drug manufacturer's name]

\section{Searching other resources}

Manual searches including scanning of reference lists in relevant papers, specialist journals and conference proceedings will be performed. Additional trials will be sought through the WHO Trial Register, ${ }^{41}$ ClinicalTrials. gov $^{42}$ and through correspondence with experts.

\section{Data collection and analysis}

Three authors (MH, DPS and KHM) will independently extract data and resolve disagreements through discussion before analysis. In the case of unresolved matters, a third party (TV, LLG and/or FKK) will be involved. When necessary data are not included in the published trial reports, authors of included trials will be contacted for additional information. Also, principal investigators of the included randomised trials will be contacted to obtain validated data based on individual patients.

\section{Selection of studies}

Trials identified through the electronic and manual searches will be listed, and-using the criteria described above-trials will be selected for inclusion.

\section{Data extraction and management}

Standardised extraction forms will be used. The following data will be extracted from included trials:

- Patient characteristics: inclusion criteria, proportion of patients with T2DM, mean age, proportion of men, BMI, baseline HbAlc, baseline fasting plasma glucose, baseline total cholesterol, LDL cholesterol,
HDL cholesterol, triglycerides and antidiabetic background treatment.

- Intervention characteristics: type, dose and duration of interventions applied.

- Trial characteristics: number of clinical sites, country of origin and funding.

\section{Assessment of risk of bias in included studies}

- Randomisation (selection bias): based on empirical evidence, the randomisation methods will be extracted as the primary measure of bias control. ${ }^{43}$ Methodological quality in the randomisation methods will be based on the allocation sequence generation (classed as adequate if based on computer-generated random numbers, a table of random numbers or similar) and allocation concealment (classed as adequate if randomisation was performed through a central independent unit, identically appearing coded drug containers, serially numbered opaque sealed envelopes or similar) and incomplete outcome data (whether all patients were accounted for).

- Blinding (performance and detection bias): we will extract data on whether single or double blinding was performed, the method of blinding (eg, use of placebo) and the persons who were blinded with regard to the interventions assessed (ie, patients, healthcare providers or other persons involved in the trial).

- Incomplete outcome data (attrition bias): the extent to which all patients lost to follow-up are accounted for will be evaluated as a measure of attrition bias.

- Outcome reporting (reporting bias): the extent to which clinically relevant outcome measures are reported and differences between trial protocols and subsequent reports will be evaluated as a marker of reporting bias.

- Other biases: sample size calculations and whether the planned sample size was achieved will be evaluated.

\section{Measures of treatment effect}

Dichotomous data will be analysed using risk differences (RD) and continuous data using weighted mean differences, both with $95 \%$ CIs. For dichotomous data, the number needed to treat will be calculated based on the $\mathrm{RD}$ as $1 / \mathrm{RD}$.

\section{Unit of analysis issues}

For crossover trials, data from the first treatment period will be used. For trials in which more than one control group was assessed, the primary analysis will combine data from each control group. Subgroup analyses on control groups will also be performed. Each patient will be counted only once in the analysis. 


\section{Dealing with missing data}

Intention-to-treat analyses including all patients randomised will be performed. In the case of patients with missing outcome data, carry forward of the last observed response will be used. Individual patient data will be sought from the original source or from the published trial reports where individual patient data are unavailable.

\section{Assessment of heterogeneity}

The intertrial heterogeneity will be expressed as $\mathrm{I}^{2}$ values.

\section{Assessment of reporting biases}

We will extract whether clinically relevant outcomes are reported and compare trial protocols with subsequent publications when available.

\section{Data analysis}

Analyses will be performed in RevMan ${ }^{44}$ and Stata V.12 (Stata Corp, Texas, USA). The primary meta-analyses will be performed using random effects models owing to an expected intertrial heterogeneity.

\section{Sequential analysis}

Sequential analyses will be performed to evaluate the robustness of the results after correction for potential errors associated with cumulative testing. The analyses will be performed using the results of the primary meta-analysis, model-based heterogeneity and an $\alpha$-value of $5 \%$ and a power of $80 \%$.

\section{Subgroup analysis and investigation of heterogeneity}

Subgroup analyses will be performed to analyse the influence of patient, intervention and trial characteristics and intertrial heterogeneity. The subgroup analyses will compare the different types of BASs. The test for subgroup differences will be calculated and the results will be presented as $\mathrm{P}$ and $\mathrm{I}^{2}$ values.

\section{Sensitivity analysis}

Fixed effect meta-analyses will be performed to evaluate the influence of small trials. Additional sensitivity analyses with exclusion of trials with unclear randomisation will also be performed.

\section{ETHICS AND DISSEMINATION}

The study will evaluate the impact of BASs on glycaemic control (HbA1c) and also assess effects on fasting plasma glucose, body weight and lipids and adverse events associated with the use of BASs in patients with T2DM, and hence possibly contribute to the clinical management of patients with T2DM. MH will draft a paper describing the systematic review and the study will be disseminated by peer-reviewed publication and conference presentation.

Contributors MH, TV, LLG and FKK participated in the conception and design of this protocol including search strategy development. MH, DPS and KHM participated in search strategy development and performed pilot searches.
LLG provided statistical advice for the design. All authors drafted and critically reviewed the manuscript and approved the final version.

Funding $\mathrm{MH}$ and FKK are supported by an unrestricted grant from the Novo Nordisk Foundation. This research did not otherwise receive specific grant from any funding agency in the public, commercial or not-for-profit sectors. No sponsor was involved in study design, and no sponsor will have authority in collection, management, analysis and interpretation of data. Writing of the report and the decision to submit the results for publication is strictly made by the authors.

Competing interests None.

Provenance and peer review Not commissioned; externally peer reviewed.

\section{REFERENCES}

1. Nauck M, Stöckmann F, Ebert R, et al. Reduced incretin effect in type 2 (non-insulin-dependent) diabetes. Diabetologia 1986;29:46-52.

2. Knop FK, Aaboe K, Vilsbøll T, et al. Impaired incretin effect and fasting hyperglucagonaemia characterizing type 2 diabetic subjects are early signs of dysmetabolism in obesity. Diabetes Obes Metab 2012;14:500-10.

3. Gaede P, Vedel P, Larsen N, et al. Multifactorial intervention and cardiovascular disease in patients with type 2 diabetes. $N$ Engl J Med 2003;348:383-93.

4. Katsuma S, Hirasawa A, Tsujimoto G. Bile acids promote glucagon-like peptide-1 secretion through TGR5 in a murine enteroendocrine cell line STC-1. Biochem Biophys Res Commun 2005;329:386-90.

5. Thomas C, Gioiello A, Noriega L, et al. TGR5-mediated bile acid sensing controls glucose homeostasis. Cell Metab 2009;10:167-77.

6. Lefebvre $\mathrm{P}$, Cariou $\mathrm{B}$, Lien $\mathrm{F}$, et al. Role of bile acids and bile acid receptors in metabolic regulation. Physiol Rev 2009;89:147-91.

7. Davidson MH, Dillon MA, Gordon B, et al. Colesevelam hydrochloride (cholestagel): a new, potent bile acid sequestrant associated with a low incidence of gastrointestinal side effects. Arch Intern Med 1999;159:1893-900.

8. Lyons D, Webster J, Fowler G, et al. Colestipol at varying dosage intervals in the treatment of moderate hypercholesterolaemia. $\mathrm{Br} \mathrm{J}$ Clin Pharmacol 1994;37:59-62.

9. Zieve FJ, Kalin MF, Schwartz SL, et al. Results of the glucose-lowering effect of WelChol study (GLOWS): a randomized, double-blind, placebo-controlled pilot study evaluating the effect of colesevelam hydrochloride on glycemic control in subjects with type 2 diabetes. Clin Ther 2007;29:74-83.

10. Bays HE, Goldberg RB, Truitt KE, et al. Colesevelam hydrochloride therapy in patients with type 2 diabetes mellitus treated with metformin: glucose and lipid effects. Arch Intern Med 2008;168:1975-83.

11. Fonseca VA, Rosenstock J, Wang AC, et al. Colesevelam $\mathrm{HCl}$ improves glycemic control and reduces LDL cholesterol in patients with inadequately controlled type 2 diabetes on sulfonylurea-based therapy. Diabetes Care 2008;31:1479-84.

12. Goldberg RB, Fonseca VA, Truitt KE, et al. Efficacy and safety of colesevelam in patients with type 2 diabetes mellitus and inadequate glycemic control receiving insulin-based therapy. Arch Intern Med 2008;168:1531-40.

13. Staels B, Kuipers F. Bile acid sequestrants and the treatment of type 2 diabetes mellitus. Drugs 2007;67:1383-92.

14. Suzuki T, Oba K, Igari $Y$, et al. Colestimide lowers plasma glucose levels and increases plasma glucagon-like PEPTIDE-1 (7-36) levels in patients with type 2 diabetes mellitus complicated by hypercholesterolemia. J Nihon Med Sch 2007;74:338-43.

15. Beysen C, Murphy EJ, Deines K, et al. Effect of bile acid sequestrants on glucose metabolism, hepatic de novo lipogenesis, and cholesterol and bile acid kinetics in type 2 diabetes: a randomised controlled study. Diabetologia 2012;55:432-42.

16. Shang $Q$, Saumoy $M$, Holst JJ, et al. Colesevelam improves insulin resistance in a diet-induced obesity (F-DIO) rat model by increasing the release of GLP-1. Am J Physiol Gastrointest Liver Physiol 2010;298:G419-24.

17. Chen L, McNulty J, Anderson D, et al. Cholestyramine reverses hyperglycemia and enhances glucose-stimulated glucagon-like peptide 1 release in Zucker diabetic fatty rats. J Pharmacol Exp Ther 2010;334:164-70.

18. Shang $Q$, Liu MK, Saumoy M, et al. The combination of colesevelam with sitagliptin enhances glycemic control in diabetic ZDF rat model. Am J Physiol Gastrointest Liver Physiol 2012;302:G815-23. 
19. Herrema $\mathrm{H}$, Meissner M, van Dijk TH, et al. Bile salt sequestration induces hepatic de novo lipogenesis through farnesoid $X$ receptor and liver $\mathrm{X}$ receptor alpha-controlled metabolic pathways in mice. Hepatology 2010;51:806-16.

20. Maruyama T, Miyamoto $\mathrm{Y}$, Nakamura T, et al. Identification of membrane-type receptor for bile acids (M-BAR). Biochem Biophys Res Commun 2002;298:714-19.

21. Thomas C, Gioiello A, Noriega L, et al. TGR5-mediated bile acid sensing controls glucose homeostasis. Cell Metab 2009;10:167-77.

22. Handelsman $Y$. Role of bile acid sequestrants in the treatment of type 2 diabetes. Diabetes Care 2011;34(Suppl 2):S244-50.

23. Vilsbøll T, Holst JJ, Knop FK. The spectrum of antidiabetic actions of GLP-1 in patients with diabetes. Best Pract Res Clin Endocrinol Metab 2009;23:453-62.

24. Brufau G, Stellaard F, Prado K, et al. Improved glycemic control with colesevelam treatment in patients with type 2 diabetes is not directly associated with changes in bile acid metabolism. Hepatology 2010;52:1455-64.

25. Garbutt JT, Kenney TJ. Effect of cholestyramine on bile acid metabolism in normal man. J Clin Invest 1972;51:2781-9.

26. Andersén E, Karlaganis G, Sjövall J. Altered bile acid profiles in duodena bile and urine in diabetic subjects. Eur J Clin Invest 1988;18:166-72.

27. WHO Diabetes. WHO. http://www.who.int/mediacentre/factsheets/ fs312/en/ (accessed 14 May 2012).

28. Gall MA, Borch-Johnsen K, Hougaard P, et al. Albuminuria and poor glycemic control predict mortality in NIDDM. Diabetes 1995;44:1303-9.

29. Agewall S, Wikstrand J, Ljungman S, et al. Usefulness of microalbuminuria in predicting cardiovascular mortality in treated hypertensive men with and without diabetes mellitus. Risk Factor Intervention Study Group. Am J Cardiol 1997;80:164-9.

30. Stratton IM, Adler AI, Neil HA, et al. Association of glycaemia with macrovascular and microvascular complications of type 2 diabetes (UKPDS 35): prospective observational study. BMJ 2000;321:405-12.

31. Duckworth WC, Abraira C, Moritz TE, et al. The duration of diabetes affects the response to intensive glucose control in type 2 subjects: the VA Diabetes Trial. J Diabetes Complicat 2011;25:355-61.

32. Zoungas S, Chalmers J, Ninomiya T, et al. Association of HbA1c levels with vascular complications and death in patients with type 2 diabetes: evidence of glycaemic thresholds. Diabetologia 2012;55:636-43.

33. Gerstein HC, Miller ME, Genuth S, et al. Long-term effects of intensive glucose lowering on cardiovascular outcomes. $N$ Engl J Med 2011;364:818-28.

34. Ray KK, Seshasai SRK, Wijesuriya S, et al. Effect of intensive control of glucose on cardiovascular outcomes and death in patients with diabetes mellitus: a meta-analysis of randomised controlled trials. Lancet 2009;373:1765-72.

35. Turnbull FM, Abraira C, Anderson RJ, et al. Intensive glucose control and macrovascular outcomes in type 2 diabetes. Diabetologia 2009;52:2288-98.

36. Cheung BMY, Ong KL, Cherny SS, et al. Diabetes prevalence and therapeutic target achievement in the United States, 1999 to 2006. Am J Med 2009;122:443-53.

37. Inzucchi SE, Bergenstal RM, Buse JB, et al. Management of hyperglycaemia in type 2 diabetes: a patient-centered approach. Position statement of the American Diabetes Association (ADA) and the European Association for the Study of Diabetes (EASD). Diabetologia 2012;55:1577-96.

38. Cochrane Handbook for Systematic Reviews of Interventions. The Cochrane Collaboration. http://www.cochrane.org/training/ cochrane-handbook (accessed 14 May 2012).

39. Moher D, Liberati A, Tetzlaff J, et al. Preferred reporting items for systematic reviews and meta-analyses: the PRISMA statement. BMJ 2009;339:b2535.

40. WHO. Definition and diagnosis of diabetes mellitus and intermediate hyperglycaemia. WHO. http://www.who.int/diabetes/publications/ diagnosis_diabetes2006/en/index.html (accessed 14 May 2012).

41. WHO. About the ICTRP search portal. WHO. http://who.int/ictrp/ search/en/ (accessed 14 May 2012).

42. http://clinicaltrials.gov/ct2/search

43. Wood L, Egger M, Gluud LL, et al. Empirical evidence of bias in treatment effect estimates in controlled trials with different interventions and outcomes: meta-epidemiological study. BMJ 2008;336:601-5.

44. Review Manager (RevMan) [Computer program]. Version 5.1. Copenhagen: The Nordic Cochrane Centre, The Cochrane Collaboration, 2011. 